\title{
NOUVELLE
}

\section{Les lymphocytes $T$ régulateurs contrôlent la croissance du poil et préviennent le développement de la pelade}

Lisa Martin ${ }^{1}$, Jean-François Nicolas ${ }^{2}$

\author{
${ }^{1}$ Master Biologie Moléculaire et Cellulaire-M2, \\ Parcours Biologie de la Peau, Université Claude Bernard \\ Lyon 1, France. \\ ${ }^{2}$ Université Lyon 1, Inserm U11ll - CIRI, Hôpitaux de Lyon, \\ France. \\ lisa.martin@etu.univ-lyonl.fr \\ jean-francois.nicolas@chu-lyon.fr
}

> Le follicule pileux est une structure résultant de l'interaction entre l'ectoderme et le mésoderme au cours de la vie embryonnaire. Ce mini-organe est divisé en trois grandes parties: une partie haute dite « infundibulum » qui forme l'ouverture folliculaire à la surface de la peau ; une partie intermédiaire, l'isthme; et une partie profonde, le bulbe pileux, qui contient des kératinocytes matriciels et des mélanocytes qui assurent la formation et la pigmentation du poil [1, 2].

Le poil joue un rôle majeur dans I'homéostasie du tissu cutané, la protection contre les UV, la thermorégulation, l'évacuation des sécrétions (sueurs et sébum), etc. [1]. Pour maintenir ces fonctions, le poil doit se régénérer. Ce mécanisme dépend de cellules souches épithéliales (HFSC pour hair follicle stem cells), et d'un cycle de trois phases régi par des acteurs moléculaire (Wnt/ $\beta$ caténine, FGF, IGF, etc.) [2]. Ce cycle débute par une phase de croissance du poil (anagène) assurée par des kératinocytes dérivant des HFSC, qui dure de 3 à 6 ans. À l'issue de cette période, le poil subit une phase de régression et involution (catagène) qui dure quelques semaines puis une phase de repos (télogène) de 2 à 6 mois, qui aboutit finalement à sa chute, lors de la repousse d'un nouveau poil $[1,2]$.

Durant la phase anagène, le poil est considéré comme un site immunitaire privilégié, caractérisé par une forte diminution de la densité des molécules de CMH-I (complexe majeur d'histocompatibilité de classe I) au niveau des kératinocytes du bulbe pileux et une production de médiateurs immunosuppresseurs (TGF- $\beta$, ACTH, etc.) [3]. Par ailleurs, le système immunitaire au niveau du follicule a une double fonction : (1) il est dans un état d'immuno-modulation prévenant toute réponse inflammatoire ou auto-immune vis-à-vis d'un autoantigène exprimé dans le follicule pileux sain $[3,4]$; (2) il participe à la régulation du cycle pilaire; en effet, selon la phase dans laquelle se trouve le poil, des acteurs de l'immunité innée et adaptative sont recrutés et sécrètent des médiateurs modulant l'avancée du cycle pilaire [5]. Cette double fonction semble reposer sur les lymphocytes $T$ régulateurs (LTreg) dont un rôle dans le processus de régénération du poil a récemment été identifié $[6,7]$. Ces LTreg sont des acteurs clés de l'immunotolérance et du contrôle des réponses inflammatoires [7]. L'altération de leur fonctionnalité est donc à l'origine du développement de pathologies auto-immunes [7]. Ainsi cette revue se focalisera sur les fonctions des LTreg dans la croissance physiologique du poil, et sur leur implication dans une pathologie auto-immune du poil, la pelade. 
LTreg et régulation du cycle pilaire Les LTreg sont des populations de LT CD4 ${ }^{+}$impliquées dans de nombreux processus : cicatrisation, immunotolérance, contrôle immunitaire, etc. [7]. Ces cellules expriment le facteur de transcription FOXP3 (Forkhead box P3), essentiel pour leur maturation. Un déficit en LTreg est associé à une altération de l'homéostasie des tissus et au développement de pathologies auto-immunes [7]. Très tôt dans la vie néonatale, des LTreg sont recrutés au niveau du follicule pileux pour établir une tolérance visà-vis des bactéries commensales de la peau $[6,7]$. De récentes études ont également montré l'implication de ces LTreg dans le cycle pilaire [6]. En effet, une accumulation de LTreg activés (CD25 $5^{+}$, CTLA $\left.-4^{+}, I \operatorname{COS}^{+}\right)$au niveau du follicule pileux télogène semble nécessaire pour la transition de la phase télogène à la phase anagène. Par ailleurs, la déplétion de LTreg apparait responsable d'un délai d'entrée en phase anagène. Ainsi, les LTreg jouent un rôle majeur dans la régénération du poil [6].

La croissance du poil dépend également de l'activation et de la différenciation d'un pool de HFSC situées au niveau du bulbe [1]. Une étude récente a révélé une accumulation préférentielle des LTreg au niveau de la niche des HFSC en phase télogène, qui favorise la prolifération et la différenciation des HFSC [6]. Par ailleurs, l'expression par les LTreg pendant la phase télogène de Jagged 1, l'un des ligands transmembranaires des récepteurs Notch, contribue à l'activation des HFSC via la voie Notch, essentielle pour l'induction de la phase anagène $[1,6]$. Ainsi, les LTreg moduleraient le devenir des HFSC par la voie Notch-Jagged 1, favorisant le renouvellement du poil [6] (Figure 1).

De ce fait, une déficience en LTreg peut être associée à un retard ou une absence de régénération du poil, et aboutir au développement de maladies autoimmunes [7-9].
Altération des LTreg et développement de la pelade

La pelade est une maladie auto-immune caractérisée par une perte de cheveux et de poils par plaque qui peut aboutir à une alopécie. Cette pathologie serait associée à une altération de la tolérance immune pendant la phase anagène, associée notamment à une production d'interféron gamma (IFN- $\gamma$ ) et à un recrutement massif de de lymphocytes $T$ cytotoxiques (LTc) auto-réactifs activés par des complexes $\mathrm{CMH}-\mathrm{I} /$ autoantigènes présents à la surface des cellules du follicule pileux $[3,8]$. L'infiltrat inflammatoire périfolliculaire perturberait alors la maturation du follicule pileux anagène, et entraînerait une entrée prématurée en phase catagène $[3,8]$.

L'absence de contrôle de l'inflammation dans cette pathologie semble être corrélée à une altération des populations de LTreg [9]. En effet, une diminution des LTreg $\left(\mathrm{CD}^{+} \mathrm{CD}^{2} 5^{+} \mathrm{FOXP3}^{+}\right)$circulants est observée chez des patients atteints de pelade,

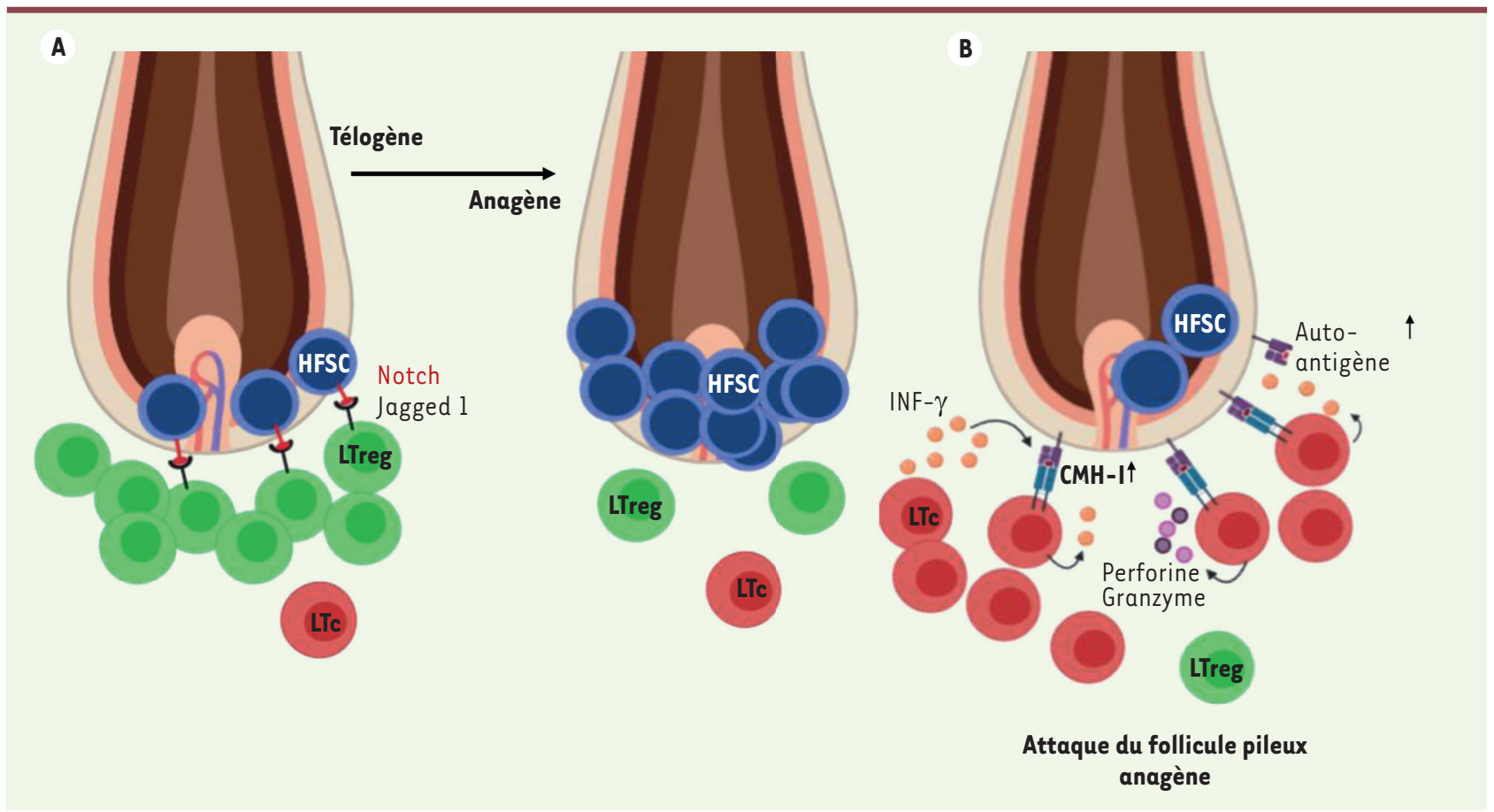

Figure 1. Les LTreg dans le processus de régénération physiologique et pathologique (pelade) du poil. (A) L'accumulation de LTreg activés exprimant Jagged 1, au niveau du follicule en phase télogène, permet l'engagement de la voie Notch dans les HFSC, assurant la transition de la phase télogène à la phase anagène. (B) L'altération de la fréquence et de la fonction des LTreg promeut l'émergence de LTc activés attaquant le follicule pileux anagène, et conduisant au développement de la pelade. 
ce qui pourrait justifier la réduction des LTreg aux sites lésionnels [9]. Par ailleurs, la fonctionnalité des LTreg serait également altérée à la suite d'une réduction de l'expression des molécules du CMH-II (HLADR) nécessaires à leur activité suppressive [9]. Ainsi, l'altération de la fréquence et de la fonction des LTreg contribuerait à l'immuno-pathologie de cette maladie en favorisant l'activité cytotoxique des LTc au follicule pileux (Figure 1).

La pelade a un réel impact sur la qualité de vie des patients (sociabilité, dépression, etc.). Il est donc nécessaire de développer des thérapies [10]. Pour leur maintien, les LTreg dépendent de certaines cytokines dont l'interleukine-2 (IL-2). Des travaux récents montrent que l'injection de faibles doses d'IL-2 favorise le recrutement de LTreg aux sites lésionnels [10]. Bien que la repousse des poils soit partielle, I'axe IL-2/LTreg constitue une nouvelle opportunité pour ces patients, mais nécessite d'être davantage étudié. Ensemble, ces observations soulignent l'importance des LTreg dans la croissance physiologique du poil et dans la prévention de la pelade [10].

\section{Conclusion}

Le follicule pileux anagène constitue un site immunitaire privilégié caractérisé par une forte diminution des molécules de CMH-I, l'expression de facteurs immunosuppresseurs, la présence de LTreg et de cellules dendritiques tolérogènes $[3,4]$. L'accumulation de LTreg activés exprimant Jagged 1 , au niveau du follicule en phase télogène permet la prolifération et la différenciation des HFSC via la voie Notch, favorisant ainsi l'entrée en phase anagène [6]. L'altération de la fréquence et de la fonction des LTreg est responsable de l'émergence de LTc affectant les kératinocytes du follicule pileux anagène, et donc la régénération du poil $[8,9]$. Néanmoins, l'utilisation de molécules pharmacologiques comme l'IL-2 pourrait restaurer les fonctions des LTreg et donc modifier le profil pathologique de la pelade.

Ainsi, les LTreg jouent un rôle crucial dans la régulation physiologique du poil [6] et dans la prévention de la pelade [10]. Cependant, les mécanismes de recrutement et d'activation des LTreg au follicule pileux télogène restent méconnus [6]. On pourrait supposer que des signaux provenant des bactéries commensales de la peau pourraient être à l'origine de la présence des LTreg au niveau du follicule en phase télogène.

L'implication des LTreg dans le processus de régénération ne s'applique pas uniquement aux poils mais également à d'autres tissus tels que la peau, le muscle squelettique et cardiaque ou encore les os [7]. Ainsi, les LTreg, sont au cœur de nombreux mécanismes et leur stimulation dans certaines pathologies, notamment auto-immunes, pourrait être bénéfique. $\diamond$ Regulatory T cells control hair growth and prevent the development of alopecia areata

\section{LIENS D'INTÉRÊT}

Les auteurs déclarent n'avoir aucun lien d'intérêt concernant les données publiées dans cet article.

\section{RÉFÉRENCES}

1. Schneider MR, Schmidt-Ullrich R, Paus R. The hair follicle as a dynamic miniorgan. Curr Biol $2009 ; 19$ : R132-42.

2. Paus $R$, Cotsarelis $G$. The biology of hair follicles. $N$ Engl J Med 1999 ; 341 : 491-7.

3. Paus R, Ito N, Takigawa M, et al. The hair follicle and immune privilege. J Invest Dermatol Symp Proc 2003 ; $8: 188-94$.

4. Azzawi S, Penzi LR, Senna MM. Immune privilege collapse and alopecia development: is stress a factor. Skin Appendage Disord 2018 ; 4 : 236-44.

5. Paus R, Christoph T, Müller-Röver $S$. Immunology of the hair follicle: a short journey into terra incognita. J Invest Dermatol Symp Proc 1999 ; 4 226-34.

6. Ali N, Zirak B, Rodriguez RS, et al. Regulatory T cells in skin facilitate epithelial stem cell differentiation. Cell 2017 ; 169 : 1119-29.el1.

7. Li J, Tan J, Martino MM, et al. Regulatory T-cells: potential regulator of tissue repair and regeneration. Front Immunol $2018 ; 9$ : 585.

8. Gilhar A, Schrum AG, Etzioni A, et al. Alopecia areata: animal models illuminate autoimmune pathogenesis and novel immunotherapeutic strategies. Autoimmun Rev 2016 ; $15: 726-35$

9. Hamed FN, Åstrand A, Bertolini M, et al. Alopecia areata patients show deficiency of $\mathrm{FOXP3} 3^{+} \mathrm{CD} 39^{+} \mathrm{T}$ regulatory cells and clonotypic restriction of Treg TCR $\beta$-chain, which highlights the immunopathological aspect of the disease. PLoS One 2019; 14 : e0210308.

10. Castela $\varepsilon$, Le Duff F, Butori $C$, et al. Effects of low-dose recombinant interleukin 2 to promote T-regulatory cells in alopecia areata. JAMA Dermatol $2014 ; 150: 748$.

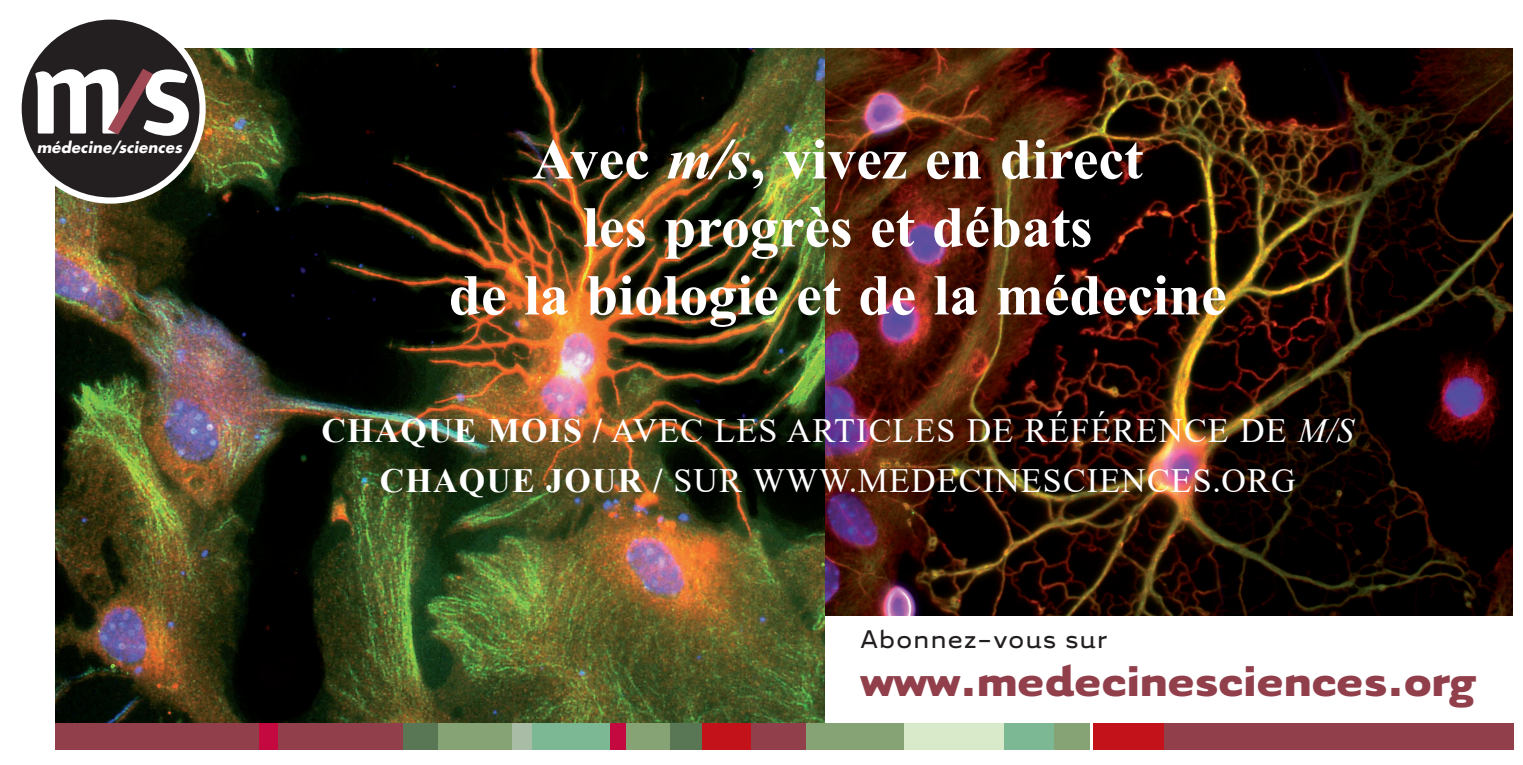

